\title{
Factors hindering critical care nurses' compliance with evidence based guidelines for prevention of secondary brain injury
}

\author{
Azza Hamdy Elssosi, Intessar Mohamed Ahmad, Alaa Mostafa Mohamed \\ Professor of Critical Care and Emergency Nursing, Critical Care \& Emergency Nursing Department, Faculty of \\ Nursing, Alexandria University Egypt. \\ Lecturer of Critical Care and Emergency Nursing, Critical Care Nursing Department, Faculty of Nursing, \\ Damanhour University Egypt. \\ Clinical Instructor, Critical Care Nursing Department, Faculty of Nursing, Damanhour, University Egypt.
}

\begin{abstract}
Background: Evidence-based clinical guidelines provide nurses with a method to use critically appraised. But, there are several barriers to their implementation which can be grouped as barriers related to individual nurse characteristics, organizational characteristics, and the nature of research information. The aim of the study: was to identify hindering factors for critical care nurses' compliance with evidence based guidelines for prevention of secondary brain injury. Materials \& Method: Two tools were developed to collect data. "Evidence based guidelines for prevention of secondary brain injury observational checklist tool" and "Factors hindering nurses' compliance with Evidenced based guidelines for prevention of secondary brain injury questionnaire tool" to determine factors hinder nurses' compliance with evidenced based guidelines for prevention of secondary brain injury. Results: The study revealed that the overall average compliance with seizure control recommendations was the highest (91.9\%) followed by maintaining adequate nutrition and preventing DVT recommendations which were more than two thirds among secondary brain injury prevention recommendations. Moreover, the organizational barriers were the most reported barriers by the majority of the CCNs (84\%). Conclusion: In conclusion, the barriers to studied nurses' compliance are ranked and categorized as organizational barriers, followed by nature of research information barriers, then personal barriers.
\end{abstract}

Key words: Hindering factors, Nursing Compliance, Evidence Based Guidelines \& Secondary Brain Injury.

\section{Introduction}

Traumatic brain injury (TBI) is a major cause of death and disability worldwide. The impact of TBI on quality of life is significant, as most suffer some degree of cognitive impairment that may include memory or motor deficits, psychological disorders, or seizures, with an increased risk for developing neurodegenerative diseases or other encephalopathies later in life. Additionally, about $1 \%$ of persons with severe TBI survive in a state of persisting unconsciousness (Tagliaferri, 2006). Understanding mechanism, classifications and pathophysiology of TBI with an integration of evidence-based practice approaches to TBI management is critical in optimizing an individual's safety, recovery and functional ability in the acute care environment (Faul, 2010).

Neurological damage does not all occur immediately at the moment of impact but evolves afterwards what is called secondary brain injury. Even though some degree of irreversible damage occurs with the primary injury, TBI is a process in which additional and progressive secondary injury evolves over the minutes, hours, and days following the primary injury. Secondary injuries occur as a complication of the primary injury and are a devastating consequence of the body's physiologic mechanisms. A significant number of those who die in the hospital have had a secondary insult that worsens the initial injury. (Bahouth, 2013).

The treatment choices for TBI patients often vary depending on the region of the country, hospital facilities, and physician and nurse experience. These various studies showed considerable variations in the care of patients with severe TBI. To improve and standardize neurocritical care management, the American Association of Neuroscience Nurses (AANN), the Brain Trauma Foundation (BTF), American Association of Neurologic Surgeons and other health care agencies have developed evidencebased guidelines (March, 2004).

Evidence-based clinical guidelines provide nurses with a method to use critically appraised and scientifically proven evidence for delivering quality health care to a specific population. Despite the potential benefits of TBI guidelines to patients and clinicians, there are several barriers to their implementation. Barriers to evidence based clinical guidelines can, be grouped as barriers related to

Vol , (4) No, (7) April 2016 
individual nurse characteristics, organizational characteristics, and the nature of research information (Ryan, 2009).

There are several personal barriers obstruct the implementation of evidence based guidelines. Nurses may lack the skill to locate research information, preferring to seek information from a colleague rather than search for a journal article or look in a textbook, the inability to critically appraise or synthesize the research literature, lack of search skills, computer skills, and library and computer access and disagreement with guidelines (Susman, 2002) \& (Ghoneim, 2012).

Organizational barriers such as lack of time for nurses to retrieve clinically useful information, inconvenient library location, lack of managerial commitment and available information technology, unavailability of training programs, lack of adequate nursing staff, insufficient resources (e.g., equipment, materials) to implement evidence based guidelines and different goals for practice between administrators and staff nurses play a vital role in hindering compliance with evidence based guidelines (Montaser, 2013). Moreover, a healthcare environment that diminishes nurses' independence, authority, or power over their practice and peer pressure to continue practices because "they have always been done this way." will decrease integration of evidence based practices.

Healthcare professionals rarely function alone; therefore, their ability to change a clinical practice cannot be made without the consent and approval of others such as hospital administrators, physicians, or other members of the healthcare team. Although a multidisciplinary focus to change will increase the success of implementation, working across disciplines also brings tension. Changing organizational culture around a practice requires that the change is consistent with organizational philosophy and political agenda and those resources are available to support the change (Sharaf, 1999)

Nature of research information can be also one of the barriers that hinder nurse's compliance with evidence based clinical guidelines. Nurses have identified problems in interpreting research findings and using them because the research is seen as too complicated, too scholarly, excessively statistical, ambiguous, and having limited or no relevance to practice. Although nurses recognize the value of research-based knowledge, they view research as overwhelming in language, volume, and writing style and want more guidance and direction for practice (Lemke, 2007).

\section{The aim of the study}

The aim of the study was to identify hindering factors for critical care nurses' compliance with evidence based guidelines for prevention of secondary brain injury.

\section{The Study questions}

- Do critical care nurses comply with evidence based guidelines for prevention of secondary brain injury?

- What are the factors that hinder critical care nurses' compliance with evidence based guidelines for prevention of secondary brain injury?

\section{Materials \& Method}

Materials

Research design

A descriptive research design was utilized to accomplish this study

\section{Setting}

This study was carried out at Alexandria Main University Hospital ICUs namely; casualty care unit (unit I), general ICU (unit III) and triage ICU. Casualty care unit (unit I) consists of two rooms with four beds each. It receives patients mainly from emergency department. Patients admitted to this unit are more acute in their condition than other units. It is mainly designed as an extended emergency or causality room. General ICU (unit III) consists of two main halls with seven to eight beds each. It receives patients who have multiple body system alterations. Triage ICU includes seven beds in the unit. It receives critically ill injured patients.

\section{Subjects}

A convenience sample of $60 \mathrm{CCN}$ nurses were included currently in the above mentioned settings and who are involved in providing direct patient care to TBI patients were recruited .

Tool: Two tools were developed and used by the researcher:

Tool One: "Evidence based guidelines for prevention of secondary brain injury observational checklist tool". It is an observational checklist designed by the researcher to gather information from critical care nurses on their compliance with evidence base guidelines for prevention of secondary brain injury. Checklist consists of 9 key items based on reviewing of the pertinent literature (Rosenfeld, 2012), (Cecil, 2011), (Kirkness, 2005) \& (Aksoy, 2000) to identify the actual nursing care provided to TBI patients. These items are rated as done correct \& complete, done correct \& incomplete, done incorrect or not done. Observational checklist includes the following items of nursing care: specific nursing interventions for maintaining adequate CPP and general maintenance nursing care for TBI patients including: (TBI patient's essential monitoring, nursing practices for oxygenation and ventilation management, ICP management, circulation and fluid management, controlling refractory ICH ?, 
seizure control, glycemic control, maintaining adequate nutrition and preventing DVT ?).

Tool Two: "Factors hindering nurses' compliance with Evidenced based guidelines for prevention of secondary brain injury questionnaire tool". This tool was developed by the researcher after reviewing the relevant literature Stein (2010) \& Davis (2000) to determine factors that may hinder nurses' compliance with evidenced based guidelines for prevention of secondary brain injury. It will include three parts:

\section{Part I: "Nurses."}

This part was used to identify nurses' related factors. It includes 9 items which are: using more traditional methods, lack of computer skills, searching skills, poor judging ability on the quality of the articles, forgetfulness, lack interest, believing that evidencebased guidelines has only limited utility, seeking information from a colleague, heavy workload .

\section{Part II: "Organizational."}

This part was used to identify organizational related factors. It include 9 items which are unavailability of training programs, insufficient resources, a library or computers are not available in the work place, inadequate nursing staff, lack of authority and support from nurse managers, absence of evidencebased guidelines mentors in health-care systems, lack of time at work place to search for, read and to apply evidence based guidelines.

\section{Part III: "type of information."}

This part was used to identify factors related to nature of information that hinder nurses compliance. It includes 7 items which are large number of statistical terms, difficult research terms, inadequacy of research information to be applied into clinical practice, evidence based information is highly complicated, too scholarly, has multiple sources and always change and updated. Each item was scored on a 5 point likert scale ranging from strongly disagree (1) to strongly agree (5).

In addition, this tool also includes Nurses' characteristics such as age, sex, level of education, and years of experience in nursing field and in ICU were assessed.

\section{Method}

- An official letter from the faculty of nursing was delivered to the hospital authorities in the Main University Hospital and approval to conduct this study was obtained after providing explanation of the aim of the study.

- Secondary brain injury prevention guidelines were developed by the researcher after determining the need and scope of guidelines. The need and scope were identified through observation of the current nursing practices performed in the clinical settings. It was found that there were no nursing contribution in the plan of management of TBI patients and there were no nursing guidelines or protocol for the management of TBI patients available in the clinical settings.

- The tool of the present study was developed after reviewing the related literature. The tools were submitted to a Jury of 7 experts in Critical Care Nursing, Medical Surgical Nursing and Anesthesia to assess clarity and content validity of the tools and all necessary modifications were done accordingly.

- Reliability of tool I and tool II were tested using Cronbach's Alpha test and results were 0.78 and 0.85 respectively.

- A pilot study was carried out on 6 nurses (10\% from the study sample) to test the clarity and applicability of the research tools and they were excluded from the study. Pilot study revealed that further modifications are not needed.

\section{Data collection}

The researcher observed nurses' practice of evidence based guidelines for prevention of secondary brain injury in the intended ICUs during morning and evening shifts over a period of four consecutive months (from October 2014 to January 2015).

The performance of specific nursing interventions for maintaining adequate CPP included TBI patient's essential monitoring, nursing practices for oxygenation and ventilation management, ICP management, circulation and fluid management, controlling refractory $\mathrm{ICH}$ and seizure control and general maintenance nursing care for TBI patients such as glycemic control, maintaining adequate nutrition and Preventing DVT were assessed by the researcher using observational checklists (Tool I) in which interventions were measured using a likert type scale of three points. A score for each item was assigned as follow: done correct \& complete was scored as 2, done correct \& incomplete was scored as 1 and done incorrect or not done was scored as zero.

The data regarding the factors hindering CCNS' compliance with evidence based guidelines for prevention of secondary brain injury were collected by the researcher using structured questionnaire during nurses' break time using Tool II. The researcher provided verbal explanation for the CCNS about the purposes of the study.

At first nurses demographic data were documented in the developed tool (Tool II) then nurses' related factors, organizational related factors and nature of research information related factors were recorded by CCNS using 5 point likert scale ranging from strongly disagree (1) to strongly agree (5).

\section{Statistical Analysis}

The raw data were coded and transformed into coding sheets. The results were checked. Then, the data were entered into the Statistical Package for Social 
Sciences (SPSS) version 18 using personal computer. Output drafts were checked against the revised coded data for typing and spelling mistakes. Finally, analysis and interpretation of data were conducted.

The following statistical measures were used:
- Descriptive statistics including frequency and distribution were used to describe different characteristics.

- Kolmogorov - Smirnov test was used to examine the normality of data distribution.

\section{Results}

Table (I): Demographic characteristics work related data of nursing staff working in the intended ICUs.

\begin{tabular}{|l|c|c|}
\hline \multicolumn{1}{|c|}{ Socio-demographic and work related data } & $\begin{array}{c}\text { No. of nurses } \\
(\mathbf{n}=\mathbf{6 0})\end{array}$ & \% \\
\hline Nurses' gender & 15 & \\
Male & 45 & 25 \\
Female & & 75 \\
\hline Nurses' age (in years) & 27 & 45 \\
20-25 yrs. & 19 & 31.7 \\
$>$ 25-30 yrs. & 14 & 23.3 \\
$>$ 30 yrs. & & \\
\hline Level of education & 13 & 21.7 \\
Technical school of nursing & 12 & 20 \\
Nursing Institute technician & 35 & 58.3 \\
Bachelor of Nursing & & \\
\hline Nurses' experience in nursing field(in years) & 21 & 35 \\
5 yrs. & 18 & 30 \\
5-10 yrs. & 21 & 35 \\
$\geq 10$ years. & & \\
\hline Nurses' experience in ICU(in years) & 32 & 53.33 \\
$\leq 5$ yrs. & 11 & 18.33 \\
5-10 yrs. & 17 & 28.33 \\
\hline 10 yrs. & & \\
\hline
\end{tabular}

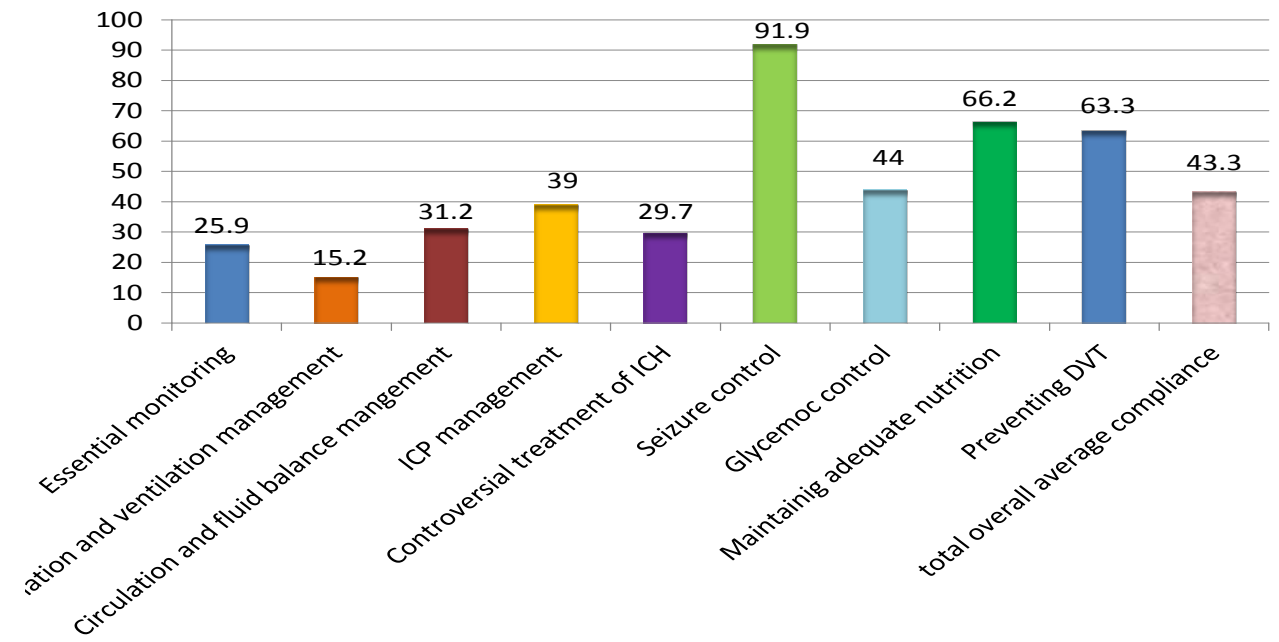

Figure (1): Nurses' compliance with evidence based guidelines recommendations for prevention of secondary brain injury. 
Table (II-a): Relation between CCNs' compliance with secondary brain injury recommendations and studied nurses' age

\begin{tabular}{|c|c|c|c|c|c|c|c|c|}
\hline \multirow{3}{*}{\multicolumn{2}{|c|}{ Recommendations }} & \multicolumn{6}{|c|}{ CCNs' age } & \multirow{4}{*}{$\begin{array}{l}\chi^{2} \mathbf{p} \\
4.50 \\
0.10\end{array}$} \\
\hline & & \multicolumn{2}{|c|}{$20-25$ years } & \multicolumn{2}{|c|}{ 25-29 Years } & \multicolumn{2}{|c|}{ More than 30 Years } & \\
\hline & & No & $\%$ & No & $\%$ & No & $\%$ & \\
\hline 1- Essential monitoring & $\begin{array}{c}\text { Done } \\
\text { Not done }\end{array}$ & $\begin{array}{l}118 \\
386\end{array}$ & $\begin{array}{l}23.4 \\
76.6\end{array}$ & $\begin{array}{l}108 \\
225\end{array}$ & $\begin{array}{l}29.8 \\
70.2\end{array}$ & $\begin{array}{c}68 \\
202\end{array}$ & $\begin{array}{l}25.2 \\
74.8\end{array}$ & \\
\hline $\begin{array}{l}\text { II- Oxygenation and } \\
\text { ventilation management }\end{array}$ & $\begin{array}{c}\text { Done } \\
\text { Not done }\end{array}$ & $\begin{array}{l}337 \\
630\end{array}$ & $\begin{array}{l}34.1 \\
65.9\end{array}$ & $\begin{array}{l}226 \\
454\end{array}$ & $\begin{array}{l}33.2 \\
66.8\end{array}$ & $\begin{array}{l}169 \\
332\end{array}$ & $\begin{array}{l}33.7 \\
66.3\end{array}$ & $\begin{array}{l}0.49 \\
0.78\end{array}$ \\
\hline $\begin{array}{l}\text { III- Circulation and fluid } \\
\text { balance management }\end{array}$ & $\begin{array}{c}\text { Done } \\
\text { Not done }\end{array}$ & $\begin{array}{c}4 \\
213\end{array}$ & $\begin{array}{c}1.8 \\
98.2\end{array}$ & $\begin{array}{c}0 \\
152\end{array}$ & $\begin{array}{c}0 \\
100\end{array}$ & $\begin{array}{c}0 \\
112\end{array}$ & $\begin{array}{c}0 \\
100\end{array}$ & $\begin{array}{l}4.90 \\
0.08\end{array}$ \\
\hline IV- ICP management & $\begin{array}{c}\text { Done } \\
\text { Not done }\end{array}$ & $\begin{array}{l}349 \\
559\end{array}$ & $\begin{array}{l}38.4 \\
61.6\end{array}$ & $\begin{array}{l}281 \\
394\end{array}$ & $\begin{array}{l}41.6 \\
58.4\end{array}$ & $\begin{array}{l}180 \\
313\end{array}$ & $\begin{array}{l}36.5 \\
63.5\end{array}$ & $\begin{array}{l}3.36 \\
0.18\end{array}$ \\
\hline $\begin{array}{l}\text { V- Controversial treatment of } \\
\text { refractory ICH }\end{array}$ & $\begin{array}{c}\text { Done } \\
\text { Not done }\end{array}$ & $\begin{array}{c}86 \\
213 \\
\end{array}$ & $\begin{array}{l}28.8 \\
71.2\end{array}$ & $\begin{array}{c}67 \\
152\end{array}$ & $\begin{array}{l}30.6 \\
69.4\end{array}$ & $\begin{array}{c}49 \\
112\end{array}$ & $\begin{array}{l}30.4 \\
69.6\end{array}$ & $\begin{array}{l}0.25 \\
0.88\end{array}$ \\
\hline VI- Seizure control & $\begin{array}{c}\text { Done } \\
\text { Not done }\end{array}$ & $\begin{array}{c}108 \\
13\end{array}$ & $\begin{array}{l}89.3 \\
10.7\end{array}$ & $\begin{array}{c}91 \\
3\end{array}$ & $\begin{array}{c}96.8 \\
3.2\end{array}$ & $\begin{array}{c}62 \\
7\end{array}$ & $\begin{array}{l}89.9 \\
10.1\end{array}$ & $\begin{array}{l}4.56 \\
0.10\end{array}$ \\
\hline VII- Glycemic control & $\begin{array}{c}\text { Done } \\
\text { Not done }\end{array}$ & $\begin{array}{l}165 \\
255\end{array}$ & $\begin{array}{l}39.3 \\
60.7\end{array}$ & $\begin{array}{l}139 \\
161\end{array}$ & $\begin{array}{l}46.3 \\
53.7\end{array}$ & $\begin{array}{l}111 \\
113\end{array}$ & $\begin{array}{l}49.6 \\
50.4\end{array}$ & $\begin{array}{l}7.25 \\
0.06\end{array}$ \\
\hline $\begin{array}{l}\text { VIII- Maintaining adequate } \\
\text { nutrition }\end{array}$ & $\begin{array}{c}\text { Done } \\
\text { Not done }\end{array}$ & $\begin{array}{c}295 \\
90\end{array}$ & $\begin{array}{l}76.6 \\
23.4\end{array}$ & $\begin{array}{c}220 \\
64\end{array}$ & $\begin{array}{l}77.5 \\
22.5\end{array}$ & $\begin{array}{c}144 \\
62\end{array}$ & $\begin{array}{l}69.9 \\
30.1\end{array}$ & $\begin{array}{l}4.30 \\
0.11\end{array}$ \\
\hline IX- Preventing DVT & $\begin{array}{c}\text { Done } \\
\text { Not done }\end{array}$ & $\begin{array}{c}129 \\
81\end{array}$ & $\begin{array}{l}61.4 \\
38.6\end{array}$ & $\begin{array}{l}99 \\
53\end{array}$ & $\begin{array}{l}65.1 \\
34.9\end{array}$ & $\begin{array}{l}72 \\
40\end{array}$ & $\begin{array}{l}64.3 \\
35.7\end{array}$ & $\begin{array}{l}0.58 \\
0.74\end{array}$ \\
\hline
\end{tabular}

$\chi^{2}:$ Chi square test

*: Statistically significant at $p \leq 0.05$

Table (II-b): Relation between CCNs' compliance with secondary brain injury recommendations and studied nurses' level of education.

\begin{tabular}{|c|c|c|c|c|c|c|c|c|}
\hline \multirow{3}{*}{\multicolumn{2}{|c|}{ Recommendations }} & \multicolumn{6}{|c|}{ CCNs' level of education } & \multirow{4}{*}{$\begin{array}{l}\chi^{2} \mathbf{p} \\
3.73 \\
0.44\end{array}$} \\
\hline & & \multicolumn{2}{|c|}{$\begin{array}{c}\text { Technical } \\
\text { school of } \\
\text { nursing }\end{array}$} & \multicolumn{2}{|c|}{$\begin{array}{c}\text { Nursing } \\
\text { Institute } \\
\text { technician }\end{array}$} & \multicolumn{2}{|c|}{$\begin{array}{c}\text { Bachelor of } \\
\text { Nursing }\end{array}$} & \\
\hline & & No & $\%$ & No & $\%$ & No & $\%$ & \\
\hline 1- Essential monitoring & $\begin{array}{l}\text { Done } \\
\text { Not done }\end{array}$ & $\begin{array}{c}76 \\
201\end{array}$ & $\begin{array}{l}27.4 \\
72.6\end{array}$ & $\begin{array}{c}56 \\
181\end{array}$ & $\begin{array}{l}23.6 \\
76.4\end{array}$ & $\begin{array}{l}162 \\
488\end{array}$ & $\begin{array}{l}24.9 \\
75.1\end{array}$ & \\
\hline $\begin{array}{l}\text { II- Oxygenation and } \\
\text { ventilation management }\end{array}$ & $\begin{array}{l}\text { Done } \\
\text { Not done }\end{array}$ & $\begin{array}{l}157 \\
309 \\
\end{array}$ & $\begin{array}{l}33.7 \\
66.3 \\
\end{array}$ & $\begin{array}{l}142 \\
286 \\
\end{array}$ & $\begin{array}{l}33.2 \\
66.8 \\
\end{array}$ & $\begin{array}{l}433 \\
821 \\
\end{array}$ & $\begin{array}{l}34.5 \\
65.5 \\
\end{array}$ & $\begin{array}{l}0.32 \\
0.98 \\
\end{array}$ \\
\hline $\begin{array}{l}\text { III- Circulation and fluid } \\
\text { balance management }\end{array}$ & $\begin{array}{l}\text { Done } \\
\text { Not done }\end{array}$ & $\begin{array}{c}0 \\
104\end{array}$ & $\begin{array}{c}0 \\
100\end{array}$ & $\begin{array}{c}1 \\
96\end{array}$ & $\begin{array}{c}1 \\
99\end{array}$ & $\begin{array}{c}3 \\
304\end{array}$ & $\begin{array}{c}1 \\
99\end{array}$ & $\begin{array}{l}1.22 \\
0.87\end{array}$ \\
\hline IV- ICP management & $\begin{array}{l}\text { Done } \\
\text { Not done }\end{array}$ & $\begin{array}{l}181 \\
275 \\
\end{array}$ & $\begin{array}{l}39.7 \\
60.3 \\
\end{array}$ & $\begin{array}{l}155 \\
266 \\
\end{array}$ & $\begin{array}{l}36.8 \\
63.2 \\
\end{array}$ & $\begin{array}{l}474 \\
725 \\
\end{array}$ & $\begin{array}{l}39.5 \\
60.5 \\
\end{array}$ & $\begin{array}{l}2.42 \\
0.65 \\
\end{array}$ \\
\hline $\begin{array}{l}\text { V- Controversial treatment of } \\
\text { refractory ICH }\end{array}$ & $\begin{array}{l}\text { Done } \\
\text { Not done }\end{array}$ & $\begin{array}{c}53 \\
104\end{array}$ & $\begin{array}{l}33.7 \\
66.3\end{array}$ & $\begin{array}{l}34 \\
96\end{array}$ & $\begin{array}{l}26.2 \\
73.8\end{array}$ & $\begin{array}{l}110 \\
277\end{array}$ & $\begin{array}{l}28.4 \\
71.6\end{array}$ & $\begin{array}{l}2.68 \\
0.61\end{array}$ \\
\hline VI- Seizure control & $\begin{array}{l}\text { Done } \\
\text { Not done }\end{array}$ & $\begin{array}{c}63 \\
1\end{array}$ & $\begin{array}{c}98.4 \\
1.6\end{array}$ & $\begin{array}{c}51 \\
8\end{array}$ & $\begin{array}{l}86.4 \\
13.6\end{array}$ & $\begin{array}{c}147 \\
14\end{array}$ & $\begin{array}{c}91.3 \\
8.7\end{array}$ & $\begin{array}{l}6.78 \\
0.14\end{array}$ \\
\hline VII- Glycemic control & $\begin{array}{l}\text { Done } \\
\text { Not done }\end{array}$ & $\begin{array}{c}95 \\
109\end{array}$ & $\begin{array}{l}46.6 \\
53.4\end{array}$ & $\begin{array}{l}94 \\
98\end{array}$ & $\begin{array}{l}49 \\
51\end{array}$ & $\begin{array}{l}226 \\
322\end{array}$ & $\begin{array}{l}41.2 \\
58.8\end{array}$ & $\begin{array}{l}2.86 \\
0.07\end{array}$ \\
\hline $\begin{array}{l}\text { VIII- Maintaining adequate } \\
\text { nutrition }\end{array}$ & $\begin{array}{l}\text { Done } \\
\text { Not done }\end{array}$ & $\begin{array}{c}135 \\
49 \\
\end{array}$ & $\begin{array}{l}73.4 \\
26.6\end{array}$ & $\begin{array}{c}139 \\
37 \\
\end{array}$ & $\begin{array}{l}79 \\
21 \\
\end{array}$ & $\begin{array}{l}385 \\
130 \\
\end{array}$ & $\begin{array}{l}74.7 \\
25.3 \\
\end{array}$ & $\begin{array}{l}5.22 \\
0.26 \\
\end{array}$ \\
\hline IX- Preventing DVT & $\begin{array}{l}\text { Done } \\
\text { Not done }\end{array}$ & $\begin{array}{l}67 \\
37\end{array}$ & $\begin{array}{l}64.5 \\
35.5\end{array}$ & $\begin{array}{l}58 \\
38\end{array}$ & $\begin{array}{l}60.4 \\
39.6\end{array}$ & $\begin{array}{c}175 \\
99\end{array}$ & $\begin{array}{l}63.8 \\
36.2\end{array}$ & $\begin{array}{l}3.57 \\
0.46\end{array}$ \\
\hline
\end{tabular}


Table (II-c): Relation between CCNs' compliance with secondary brain injury recommendation and studied nurses' experience in nursing field.

\begin{tabular}{|c|c|c|c|c|c|c|c|c|}
\hline \multirow{3}{*}{\multicolumn{2}{|c|}{ Recommendations }} & \multicolumn{6}{|c|}{ CCNs' Experience in nursing field } & \multirow{3}{*}{$\chi^{2} \mathbf{p}$} \\
\hline & & \multicolumn{2}{|c|}{$<5$ years } & \multicolumn{2}{|c|}{ 5-10 years } & \multicolumn{2}{|c|}{$\begin{array}{c}\text { More than } 10 \\
\text { years }\end{array}$} & \\
\hline & & No & $\%$ & No & $\%$ & No & $\%$ & \\
\hline 1- Essential monitoring & $\begin{array}{l}\text { Done } \\
\text { Not done }\end{array}$ & $\begin{array}{c}99 \\
303 \\
\end{array}$ & $\begin{array}{l}24.6 \\
75.4 \\
\end{array}$ & $\begin{array}{c}90 \\
237 \\
\end{array}$ & $\begin{array}{l}27.5 \\
72.5\end{array}$ & $\begin{array}{l}105 \\
303 \\
\end{array}$ & $\begin{array}{l}25.7 \\
74.3\end{array}$ & $\begin{array}{l}0.79 \\
0.67\end{array}$ \\
\hline $\begin{array}{l}\text { II- Oxygenation and } \\
\text { ventilation management }\end{array}$ & $\begin{array}{l}\text { Done } \\
\text { Not done }\end{array}$ & $\begin{array}{l}266 \\
489 \\
\end{array}$ & $\begin{array}{l}35.2 \\
64.8 \\
\end{array}$ & $\begin{array}{l}211 \\
429 \\
\end{array}$ & $\begin{array}{l}33 \\
67 \\
\end{array}$ & $\begin{array}{l}255 \\
498 \\
\end{array}$ & $\begin{array}{l}33.9 \\
66.1 \\
\end{array}$ & $\begin{array}{l}0.81 \\
0.66\end{array}$ \\
\hline $\begin{array}{l}\text { III- Circulation and fluid } \\
\text { balance management }\end{array}$ & $\begin{array}{l}\text { Done } \\
\text { Not done } \\
\end{array}$ & $\begin{array}{c}1 \\
168 \\
\end{array}$ & $\begin{array}{c}0.6 \\
99.4 \\
\end{array}$ & $\begin{array}{c}3 \\
141 \\
\end{array}$ & $\begin{array}{c}2.1 \\
97.9 \\
\end{array}$ & $\begin{array}{c}0 \\
0 \\
168 \\
\end{array}$ & $\begin{array}{c}0 \\
100 \\
\end{array}$ & $\begin{array}{l}4.26 \\
0.11 \\
\end{array}$ \\
\hline IV- ICP management & $\begin{array}{l}\text { Done } \\
\text { Not done } \\
\end{array}$ & $\begin{array}{l}285 \\
438 \\
\end{array}$ & $\begin{array}{l}39.4 \\
60.6 \\
\end{array}$ & $\begin{array}{l}246 \\
369 \\
\end{array}$ & $\begin{array}{l}40 \\
60 \\
\end{array}$ & $\begin{array}{l}279 \\
459 \\
\end{array}$ & $\begin{array}{l}37.8 \\
62.2 \\
\end{array}$ & $\begin{array}{l}0.75 \\
0.68 \\
\end{array}$ \\
\hline $\begin{array}{l}\text { V-Controversial treatment of } \\
\text { refractory ICH }\end{array}$ & $\begin{array}{l}\text { Done } \\
\text { Not done }\end{array}$ & $\begin{array}{c}68 \\
168 \\
\end{array}$ & $\begin{array}{l}28.8 \\
71.2\end{array}$ & $\begin{array}{c}52 \\
141 \\
\end{array}$ & $\begin{array}{l}26.9 \\
73.1 \\
\end{array}$ & $\begin{array}{c}82 \\
168 \\
\end{array}$ & $\begin{array}{l}32.8 \\
67.2 \\
\end{array}$ & $\begin{array}{l}1.93 \\
0.37\end{array}$ \\
\hline VI- Seizure control & $\begin{array}{l}\text { Done } \\
\text { Not done }\end{array}$ & $\begin{array}{l}92 \\
10 \\
\end{array}$ & $\begin{array}{c}90.2 \\
9.8 \\
\end{array}$ & $\begin{array}{c}73 \\
4 \\
\end{array}$ & $\begin{array}{c}94.8 \\
5.2 \\
\end{array}$ & $\begin{array}{c}96 \\
9\end{array}$ & $\begin{array}{c}91.4 \\
8.6\end{array}$ & $\begin{array}{l}1.30 \\
0.52\end{array}$ \\
\hline VII- Glycemic control & $\begin{array}{l}\text { Done } \\
\text { Not done } \\
\end{array}$ & $\begin{array}{l}133 \\
203 \\
\end{array}$ & $\begin{array}{l}39.6 \\
60.4 \\
\end{array}$ & $\begin{array}{l}120 \\
152 \\
\end{array}$ & $\begin{array}{l}44.1 \\
55.9 \\
\end{array}$ & $\begin{array}{l}162 \\
174 \\
\end{array}$ & $\begin{array}{l}48.2 \\
51.8 \\
\end{array}$ & $\begin{array}{l}5.08 \\
0.07 \\
\end{array}$ \\
\hline $\begin{array}{l}\text { VIII- Maintaining adequate } \\
\text { nutrition }\end{array}$ & $\begin{array}{l}\text { Done } \\
\text { Not done }\end{array}$ & $\begin{array}{c}235 \\
71 \\
\end{array}$ & $\begin{array}{l}76.8 \\
23.2 \\
\end{array}$ & $\begin{array}{c}202 \\
60 \\
\end{array}$ & $\begin{array}{l}77.1 \\
22.9 \\
\end{array}$ & $\begin{array}{c}222 \\
85\end{array}$ & $\begin{array}{l}72.3 \\
27.7\end{array}$ & $\begin{array}{l}2.29 \\
0.31 \\
\end{array}$ \\
\hline IX- Preventing DVT & $\begin{array}{l}\text { Done } \\
\text { Not done }\end{array}$ & $\begin{array}{c}106 \\
62\end{array}$ & $\begin{array}{l}63.1 \\
36.9\end{array}$ & $\begin{array}{l}87 \\
51\end{array}$ & $\begin{array}{l}63 \\
37 \\
\end{array}$ & $\begin{array}{c}107 \\
61\end{array}$ & $\begin{array}{l}63.7 \\
36.3\end{array}$ & $\begin{array}{l}0.99 \\
0.06\end{array}$ \\
\hline
\end{tabular}

$\chi^{2}:$ Chi square test

*. Statistically significant at $p \leq 0.05$

Table (II-d): Relation between CCNs' compliance with secondary brain injury recommendations and studied nurses' ICU experience

\begin{tabular}{|c|c|c|c|c|c|c|c|c|}
\hline \multirow{3}{*}{\multicolumn{2}{|c|}{ Recommendations }} & \multicolumn{6}{|c|}{ CCNs' ICU Experience } & \multirow{4}{*}{$\begin{array}{c}\chi^{2} \mathbf{p} \\
0.89 \\
0.63\end{array}$} \\
\hline & & \multicolumn{2}{|c|}{$<5$ years } & \multicolumn{2}{|c|}{ 5-10 years } & \multicolumn{2}{|c|}{ More than 10 years } & \\
\hline & & No & $\%$ & No & $\%$ & No & $\%$ & \\
\hline 1- Essential monitoring & $\begin{array}{l}\text { Done } \\
\text { Not done } \\
\end{array}$ & $\begin{array}{l}150 \\
448 \\
\end{array}$ & $\begin{array}{l}25.1 \\
74.9 \\
\end{array}$ & $\begin{array}{c}51 \\
153 \\
\end{array}$ & $\begin{array}{l}25 \\
75 \\
\end{array}$ & $\begin{array}{c}93 \\
242 \\
\end{array}$ & $\begin{array}{l}27.8 \\
72.2 \\
\end{array}$ & \\
\hline $\begin{array}{l}\text { II- Oxygenation and } \\
\text { ventilation management }\end{array}$ & $\begin{array}{l}\text { Done } \\
\text { Not done }\end{array}$ & $\begin{array}{l}395 \\
750\end{array}$ & $\begin{array}{l}34.5 \\
65.5\end{array}$ & $\begin{array}{l}131 \\
263\end{array}$ & $\begin{array}{l}33.2 \\
66.8\end{array}$ & $\begin{array}{l}206 \\
403\end{array}$ & $\begin{array}{l}33.8 \\
66.2\end{array}$ & $\begin{array}{l}0.22 \\
0.89\end{array}$ \\
\hline $\begin{array}{l}\text { III- Circulation and fluid } \\
\text { balance management }\end{array}$ & $\begin{array}{l}\text { Done } \\
\text { Not done }\end{array}$ & $\begin{array}{c}4 \\
253 \\
\end{array}$ & $\begin{array}{c}1.6 \\
98.4 \\
\end{array}$ & $\begin{array}{c}0 \\
88 \\
\end{array}$ & $\begin{array}{c}0 \\
100 \\
\end{array}$ & $\begin{array}{c}0 \\
136 \\
\end{array}$ & $\begin{array}{c}0 \\
100 \\
\end{array}$ & $\begin{array}{l}3.51 \\
0.17\end{array}$ \\
\hline IV- ICP management & $\begin{array}{l}\text { Done } \\
\text { Not done }\end{array}$ & $\begin{array}{l}438 \\
650 \\
\end{array}$ & $\begin{array}{l}40.3 \\
59.7 \\
\end{array}$ & $\begin{array}{l}150 \\
241 \\
\end{array}$ & $\begin{array}{l}38.4 \\
61.6 \\
\end{array}$ & $\begin{array}{l}222 \\
375 \\
\end{array}$ & $\begin{array}{l}37.2 \\
62.8 \\
\end{array}$ & $\begin{array}{l}1.61 \\
0.44 \\
\end{array}$ \\
\hline $\begin{array}{l}\text { V-Controversial treatment of } \\
\text { refractory ICH }\end{array}$ & $\begin{array}{l}\text { Done } \\
\text { Not done }\end{array}$ & $\begin{array}{c}93 \\
253\end{array}$ & $\begin{array}{l}26.9 \\
73.1\end{array}$ & $\begin{array}{l}43 \\
88\end{array}$ & $\begin{array}{l}32.8 \\
67.2\end{array}$ & $\begin{array}{c}66 \\
136\end{array}$ & $\begin{array}{l}32.7 \\
67.3\end{array}$ & $\begin{array}{l}2.78 \\
0.24\end{array}$ \\
\hline VI- Seizure control & $\begin{array}{l}\text { Done } \\
\text { Not done }\end{array}$ & $\begin{array}{c}133 \\
13 \\
\end{array}$ & $\begin{array}{c}91.1 \\
8.9\end{array}$ & $\begin{array}{c}46 \\
4\end{array}$ & $\begin{array}{c}92 \\
8\end{array}$ & $\begin{array}{c}82 \\
6 \\
\end{array}$ & $\begin{array}{c}93.2 \\
6.8\end{array}$ & $\begin{array}{l}0.32 \\
0.85\end{array}$ \\
\hline VII- Glycemic control & $\begin{array}{l}\text { Done } \\
\text { Not done }\end{array}$ & $\begin{array}{l}201 \\
299 \\
\end{array}$ & $\begin{array}{l}40.2 \\
59.8 \\
\end{array}$ & $\begin{array}{l}80 \\
92 \\
\end{array}$ & $\begin{array}{l}46.5 \\
53.5 \\
\end{array}$ & $\begin{array}{l}134 \\
138 \\
\end{array}$ & $\begin{array}{l}49.3 \\
50.7 \\
\end{array}$ & $\begin{array}{l}6.43 \\
0.08 \\
\end{array}$ \\
\hline $\begin{array}{l}\text { VIII- Maintaining adequate } \\
\text { nutrition }\end{array}$ & $\begin{array}{l}\text { Done } \\
\text { Not done }\end{array}$ & $\begin{array}{l}359 \\
107 \\
\end{array}$ & $\begin{array}{l}77 \\
23 \\
\end{array}$ & $\begin{array}{c}124 \\
38 \\
\end{array}$ & $\begin{array}{l}76.5 \\
23.5 \\
\end{array}$ & $\begin{array}{c}176 \\
71 \\
\end{array}$ & $\begin{array}{l}71.3 \\
28.7 \\
\end{array}$ & $\begin{array}{l}3.06 \\
0.21 \\
\end{array}$ \\
\hline IX- Preventing DVT & $\begin{array}{l}\text { Done } \\
\text { Not done }\end{array}$ & $\begin{array}{c}161 \\
89\end{array}$ & $\begin{array}{l}64.4 \\
35.6\end{array}$ & $\begin{array}{l}54 \\
34\end{array}$ & $\begin{array}{l}61.4 \\
38.6\end{array}$ & $\begin{array}{l}85 \\
51\end{array}$ & $\begin{array}{l}62.5 \\
37.5\end{array}$ & $\begin{array}{l}0.31 \\
0.85\end{array}$ \\
\hline
\end{tabular}

$\chi^{2}:$ Chi square test

*: Statistically significant at $p \leq 0.05$ 
Table (III): CCNs ' self-reported factors hindering their compliance with evidence based guidelines for prevention of secondary brain injury

\begin{tabular}{|c|c|c|c|c|c|c|}
\hline \multirow{2}{*}{ Hindering factors } & \multicolumn{2}{|c|}{ Disagree } & \multicolumn{2}{|c|}{ Neutral } & \multicolumn{2}{|c|}{ Agree } \\
\hline & No & $\%$ & No & $\%$ & No & $\%$ \\
\hline $\begin{array}{l}\text { Personal factors } \\
\text { Too high workload to keep up to date. }\end{array}$ & 9 & 15 & 6 & 10 & 45 & 75 \\
\hline Difficulty in judging the quality of the articles & 10 & 16.7 & 11 & 18.3 & 39 & 65 \\
\hline Limited utility of evidence-based guidelines & 10 & 16.7 & 19 & 31.7 & 31 & 51.6 \\
\hline Lack of computer skills & 23 & 38.3 & 11 & 18.3 & 26 & 43.3 \\
\hline Lack of websites searching skills & 23 & 38.3 & 11 & 18.3 & 26 & 43.3 \\
\hline Seeking information from a colleague & 24 & 40 & 10 & 16.7 & 26 & 43.3 \\
\hline Using more traditional methods & 30 & 50 & 6 & 10 & 24 & 40 \\
\hline Forgetfulness & 30 & 50 & 11 & 18.3 & 19 & 31.6 \\
\hline Lack of interest & 40 & 66.7 & 8 & 13.3 & 12 & 20 \\
\hline $\begin{array}{l}\text { Nature of research information factors } \\
\text { Continuous changing and updating }\end{array}$ & 4 & 6.7 & 6 & 10 & 50 & 83.3 \\
\hline Not complied in one place & 5 & 8.3 & 11 & 18.3 & 44 & 73.4 \\
\hline Too complicated & 6 & 10 & 17 & 28.3 & 37 & 61.7 \\
\hline Inadequate to be applied into practice & 8 & 13.3 & 16 & 26.7 & 36 & 60 \\
\hline Excessively statistical & 9 & 15 & 16 & 26.7 & 35 & 58.3 \\
\hline Too scholarly and not applicable & 6 & 10 & 22 & 36.7 & 32 & 53.3 \\
\hline Difficult research terms & 18 & 30 & 12 & 20 & 30 & 50 \\
\hline Organizational factors & & & & & & \\
\hline $\begin{array}{l}\text { Lack of time at work place to search for or read } \\
\text { evidence based guidelines }\end{array}$ & 1 & 1.7 & 1 & 1.7 & 58 & 96.6 \\
\hline Lack of a library or computers in the work place & 1 & 1.7 & 2 & 3.3 & 57 & 95 \\
\hline Shortages of nursing staff & 1 & 1.7 & 3 & 5 & 56 & 93.3 \\
\hline Unavailability of training programs & 3 & 5 & 3 & 5 & 54 & 90 \\
\hline lack of evidence-based guidelines mentors & 2 & 3.3 & 8 & 13.3 & 50 & 83.3 \\
\hline Insufficient resources & 8 & 13.3 & 6 & 10 & 46 & 76.7 \\
\hline lack of nurse managers' support & 9 & 15 & 8 & 13.3 & 43 & 71.7 \\
\hline Lack of authority & 8 & 13.3 & 11 & 18.3 & 41 & 68.3 \\
\hline
\end{tabular}

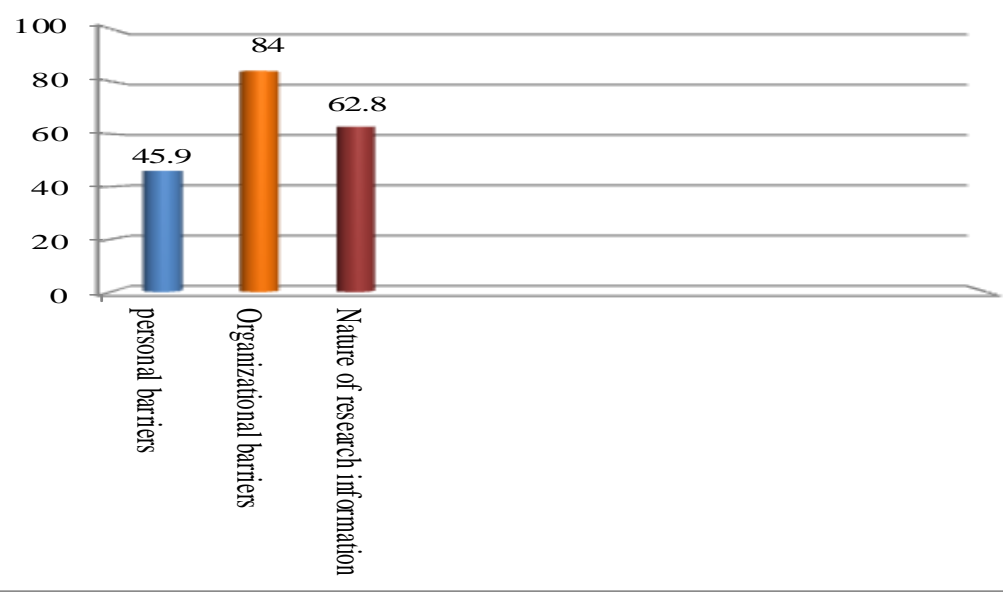

Figure (2): distribution of barriers to CCNs compliance with secondary brain injury preventions guidelines. 
Table (I): Illustrates socio-demographic characteristics of nursing staff working in the intended ICUs. The total number of nursing staff who provide direct care for TBI patient in the intended ICUs was 60 nurses. Concerning nurses' gender, this table shows that three fourths of studied nurses $(75 \%)$ were females. The age of the studied nurses ranged between 21 and 38 years with $45 \%$ of them had 25 years or less. Regarding the level of education, it can be noted from this table that more than one half of studied nurses $(58.3 \%)$ holds a baccalaureate degree. As regards the studied nurses' years of experience in ICU, this table depicts that more than half of studied nurses (53.33) had experience less than or equal 5 years.

Figure (1): Shows nurses' compliance with evidence based guidelines recommendations for prevention of secondary brain injury. It can be noted from this figure that the lowest overall average compliance $(15.2 \%)$ was for oxygenation and ventilation management recommendations followed by the overall compliance with essential monitoring recommendations and controversial treatment of $\mathrm{ICH}$ recommendations which represents $25.9 \%$ and $29.7 \%$ respectively. It can also be noted from this figure that the overall average compliance with seizure control recommendations was the highest $(91.9 \%)$ among secondary brain injury prevention recommendations.

Table (II a-d): Reveal that there is no relation between CCNs' compliance with secondary brain injury recommendations and the characteristics of the studied nurses'

Table (III): Reveals that the most frequently reported three personal barriers were; too high nurses' workload to keep up to date with all new evidence $(75 \%)$; difficulty in judging the quality of the research articles by the nurses (65\%); and nurses' perception that evidence based guidelines have only limited utility $(51.6 \%)$. It can be noted from this table that the most frequently $(96.6 \%)$ reported organizational barrier by the CCNs was lack of time at workplace to search for or read research articles, followed by lack of a library or computers at the workplace which was reported by $95 \%$ of the studied nurses. Moreover, it can also be noted from the same table that the third organizational barrier reported by $93.3 \%$ of the studied nurses was shortages of nursing staff.

This table illustrates that continuous updating and changing of research information was the first reported barrier by the majority of the studied nurses $(83.3 \%)$. It can also be noted from this table that near three quarters $(73.4 \%)$ of the $\mathrm{CCNs}$ ranked research information not complied in one place as the second barriers. Additionally, this table also shows that the third barriers related to nature of research information was that research information is too complicated, this barrier was reported by more than two thirds $(61.7 \%)$ of the studied nurses.

Figure (2): shows distribution of barriers to $\mathrm{CCNs}$ compliance with secondary brain injury preventions guidelines. It can be detected from this figure that the organizational barriers were the most reported barriers by the majority of the CCNs (84\%). While, less than one half of the CCNs $(45.9 \%)$ reported that; personal barriers hindering their compliance with secondary brain injury preventions guidelines.

\section{Discussion}

Secondary brain injury is associated with a significant increase in morbidity, mortality, length of stay, medical cost, and it is the leading cause of inhospital deaths after traumatic brain injury. Although the rates of secondary brain injury are high, they can be prevented and reduced as most secondary brain injuries are often the result of events that occur in the ICU (Ladanyi, 2008). Clinical practice guidelines for prevention of secondary brain injury help CCNs in decision making, improve standards of nursing care, and reduce inappropriate variations in care provided to TBI patients.

About three fourths of nurses in this study were females; middle aged less than thirty years old. The results revealed that more than a half of $\mathrm{CCNs}$ hold a baccalaureate degree while others have either diploma or technical degree (table I). It was recommended previously by both the Australian Health Advisory Committee and the Australian College of Critical Care Nurses that a minimum of $50 \%$ of nursing staff employed within in ICU should hold postgraduate qualification in their area of practices (Morton, 2009) .

American Association of Neurological Surgeons Guidelines (2007), Brighton \& Sussex University Hospitals guidline (2009) \& Thompson guidline (2011) emphasized the role of the CCNs in the management of TBI patients and prevention of secondary brain injury through compliance with prevention of secondary brain injury guidelines that encompass compliance with recommendations for essential monitoring, oxygenation and ventilation management, circulation and fluid balance management, ICP management, controversial treatment of refractory $\mathrm{ICH}$, seizure control, glycemic control, maintaining adequate nutrition, and preventing DVT.

The overall level of CCNs' compliance with prevention of secondary brain injury guidelines in the present study was less than one half, which is considered low. Low level of CCNs' compliance with prevention of secondary brain injury guidelines may 
be attributed to lack of formal standards and guidelines for prevention of secondary brain injury, lack of knowledge about poor patients' outcome associated with secondary brain injury as a result of lack of in service training programs in the intended ICUs. It may be also attributed to lack of nurses' technical skills, lack of nurses' experience, lack of educational opportunities available for CCNs including up-to-date scientific journals and books, scientific meetings, and expert nurses' supervisors for help and guidance. (Selassie, 2008) \& (Hesdorffer 2007).

It was found in previous study that the overall level of CCNs' compliance with prevention of secondary brain injury guidelines was less than one half, which is considered low Thompson (2011). Causer (2011), McNett (2010) \& Holleman (2006) emphasized the role of the CCNs in the management of TBI patients and prevention of secondary brain injury through compliance with prevention of secondary brain injury guidelines that encompass compliance with recommendations for essential monitoring, oxygenation and ventilation management, circulation and fluid balance management, ICP management, controversial treatment of refractory $\mathrm{ICH}$, seizure control, glycemic control, maintaining adequate nutrition, and preventing DVT. Therefore, the current study was conducted to identify obstructive barriers for their compliance with these guidelines.

The findings of the present study are higher than reported by McNett (2010). Who found that the percentage of the nurses' compliance with prevention of secondary brain injury guidelines was nearly less than one third and higher than this percentages reported by Hesdorffer (2002) who found that the overall level of compliance of 433 participating trauma centers admitted patients with severe TBI to their hospital in-patient units was slightly more than one third. In contrast, the percentage in the present study is lower than those reported by Wang (2013), Selassie (2008)\& Damkliang, (2013) who conducted a prospective observational study to compare Thai emergency nurses' clinical management of patients with severe TBI with best available evidence. They reported that the overall compliance of the CCNs was approximately two thirds. Similarly, Hesdorffer, (2007) found that the overall level of compliance of 413trauma centers in the United States was more than two thirds.

It was found that the top 3 personal barriers indicated by the respondents are: too high workload to keep up to date with all new evidence, difficulty in judging the quality of articles, and feeling that evidence based guidelines has only limited utility (table II). Concerning too high workload to keep up to date with all new evidence, it was reported by approximately three quarters of the CCNs and it is the most frequent reported personal barrier. This finding is supported by McCaughan, (2002) it was found that high nurses' workload is a great barrier blocks on research use. Lower percentage reported by Majid, (2011) it was cited that approximately one fifth of the nurses reported their high workload as a personal barrier that prevents them from being up to date with the new evidence.

Regarding difficulty in judging the quality of articles, the present study revealed that more than two thirds of the subjects reported their inability to judge the quality of articles. In accordance to that, Glacken, (2004) it was cited that approximately three quarters of the nurses do not feel capable of evaluating the quality of the research. In contrast, Tan, (2012) it was concluded that only less than one fifth of the study subjects do not feel capable of evaluating the quality of the research.

Additionally, the present study revealed that more than one half of the CCNs believe that evidence based guidelines has only limited utility. This finding is similar to, Oh, (2008) who reported that more than one half of the CCNs feel the benefits of changing practice will be minimal. On the contrary, the result of the current study is dissimilar to Majid, (2011) who cited that less than one quarter of the $\mathrm{CCN}$ 's believe that evidence based guidelines has only limited utility.

The finding of this study regarding personal barriers may be attributed to poor time management, lack of knowledge regarding the importance of translating evidence based guidelines into practice, and poor critical thinking. These are also may be related to another barrier which is shortage of staff nurses that hinder the implementation of new evidences.

The current study recorded that the top 3 organizational barriers indicated by the respondents are lack of time at work place to search for or read evidence based guidelines, lack of a library or computers in the work place, and inadequate nursing staff (table II). It was found that lack of time at work place to search for or read evidence based guidelines is the most frequent reported organizational barrier by the majority of the CCNs. This result is in the same line with Mehrdad, (2008) who found that lack of time at work place to or read research was the most frequent reported barrier to research utilization by the majority of the Iranian nurses. In the contrary to the finding of Chan, (2011) who cited that only one third of the emergency nurses reported the lack of time to read research at the work place as an organizational barrier.

As regards lack of library or internet access in the work place, Our finding is supported by 
Al- Ghabeesh (2014) it was found that unavailability of internet access in the unit where the nurses work is a major organizational barrier reported by the majority of the study subjects. The present study ranked lack of nursing staff as the third organizational barrier reported by more than ninety percent of the study subjects. This finding is in line with Al- Ghabeesh (2014) who ranked shortage of staff nurses as fifth organizational barrier that reported by the majority of nurses in Jordan. In contrast, the percentage in the present study is higher than those reported by two previous studies Gale (2009) in United States and Tsai (2000) in China found that lack of staff reported as an organizational barrier by slightly more than one half of the nurses.

The findings of this study can be explained as the sitting in the current study has limited financial and human resources. First, the limitation of the financial resources lead to absence of financial support to fund accessing full-text articles or even a library containing up to dates textbooks. Second, the shortage of the staff members may limit nursing staff time to read research findings.

The present study revealed that the top 3 barriers related to nature of research information reported by the CCNs were: always updated and changed, not complied in one place, and too complicated and overwhelming. The current study revealed that approximately three quarters of the respondents cited that research information is not complied in one place. This finding is consistent to Uysal (2010) it found that more than three quarters of the nurses accepted that research information is not complied in one place as a barrier to research utilization. But, in opposition to Tan (2012) who cited that only less than one quarter of nurses in Eastern Turkey reported it as a barrier to their research utilization.

The third barrier related to nature of research information is that the research information is too complicated and overwhelming. It was cited by about two thirds of the study subjects. Near percentage was reported by Uysal (2010) who concluded that more than one half of the nurses reported that the amount of research information is overwhelming. In contrast, Chan (2011) cited that only one third of emergency nurses considered the research information is too complicated and overwhelming.

The finding of this study can be explained by lack of research knowledge among the CCNs in the intended ICUs and that their years of work and experience were without any evidence of participation in research activities or training programs concerning with translating evidence based guidelines into practice. In addition, the inclusion of old nurses who did not receive research courses during their education.

\section{Conclusion}

It can be concluded that the majority of the highly ranked barriers to studied nurses' compliance with these guidelines are categorized as organizational barriers, followed by nature of research information barriers, then personal barriers. The greatest perceived barriers by $\mathrm{CCNs}$ are lack of time at work place to read research, absence of a library or computer at the work place, and shortages of nursing staff.

\section{Recommendations}

Based on the results of the present study, the following recommendations are suggested:

Recommendations regarding clinical practice

- Nursing management protocol for TBI should be applied in clinical practice as a routine of unit care.

- Strategies for updating nurses' knowledge and enhancing their practice should be developed.

- Strategies to overcome nursing shortage and increased nursing workload including part time, partial shifts, and other strategies should bed.

- Evidence-based strategies to facilitate nurses' adherence to evidence based guidelines including using checklists should be implemented.

Recommendations regarding education \& training

- Nursing students' curriculum should be focused on the vital role of the nurses who caring for TBI patients in the prevention of secondary brain injury using the best available evidence based guidelines.

- The teaching and training programs about secondary brain injury prevention guidelines should be performed through workshop, seminars, conferences, group discussion, up to date scientific journals, books, and posters.

Recommendations regarding administration

- Hospital budget should be directed to provide the needed equipment and supplies for application of secondary brain injury prevention guidelines.

- Administrators should check regularly the adherence of CCNs regarding implementation of the secondary brain injury prevention guidelines.

- Evidence based guidelines mentors should be present in health-care systems

\section{Recommendations regarding research}

- Further studies are needed to evaluate the cost effectiveness of implementation of secondary brain injury prevention guidelines.

- Studies with large sample size are needed to test the effect of implementing secondary brain injury prevention guidelines.

- Further researches are recommended to evaluate which teaching method and curriculum content are most effective to educate nurses caring for TBI 
patients and to identify barriers to incorporating this knowledge in practice.

\section{References}

1. Aksoy G., Celik S., Akyolcu N., (2000): Nursing role on preventing secondary brain injury, Accident and emergency nursing, vol 412, No (2) Pp.94-8.

2. Al-Ghabeesh S., Abu-Moghli F., Suleiman K., (2014): Predictors of Research Utilization Among Jordanian Registered Nurses: A Descriptive Correlational Study, International Journal of Medicine and Medical Sciences, $\operatorname{vol}(47)$ No(1) Pp. 1530-8.

3. American Association of Neurological Surgeons, Brain Trauma Foundation, The Joint Section on Neurotrauma of Critical Care. Guidelines for the management of severe traumatic brain injury (2007): Journal of neurotrauma, vol 24, N0(9) Pp.S1-S106.

4. Brighton and Sussex University Hospitals NHS Trust (2009): Guidelines for the management of the patient with severe traumatic brain injury.Online http: wwwbsuh $<$ b $>$ nhs $</$ b $>$ uk/EasysiteWeb/getresour ceaxd?AssetID $=54333</$ span $>$

5. Bahouth M., Yarbrough K., Patient management: nervous system In: Morton P., Fontaine D., (2013): Critical Care Nursing: A Holistic Approach. 10 ${ }^{\text {th }}$ ed. Philadelphia: PA Lippincott Wilkins, 2013;P 744-61.

6. Cecil S., Chen P., Callaway S., Rowland S., Adler D., Chen J., (2011):Traumatic brain injury: advanced multimodal neuromonitoring from theory to clinical practice, Critical care nurse, vol 31, No (2) Pp.25-36.

7. Causer T., Zipf J., George N., (2011): Protecting the Penumbra: The First Line of Defense in Preventing Secondary Brain Injury Is the Critical Care Bedside Nurse, Journal of Trauma Nursing, vol 18, No (4) Pp.104-14.

8. Chan G., Barnason S., Dakin C., Gillespie G., Kamienski M., Stapleton S., (2011): Barriers and perceived needs for understanding and using research among emergency nurses, Journal of emergency nursing, vol (37), No(1) Pp.24-31.

9. Davis A., (2000): Mechanisms of traumatic brain injury: biomechanical, structural and cellular considerations. Crit Care Nurs Q, vol (23), No(5) Pp.1-13.

10. Damkliang J., Considine J., Kent B., (2013): Thai emergency nurses' management of patients with severe traumatic brain injury: comparison of knowledge and clinical management with best available evidence, Australasian emergency nursing journal, vol (16), No (4) Pp.127-35.

11. Faul M., Xu L., Wald M., Coronado V., Traumatic Brain Injury in the United States: Emergency Department Visits, Hospitalizations and Deaths (2002-2006): Atlanta, GA: Centers for Disease Control and Prevention, National Center for Injury Preventionand Control. 2010).

12. Gale B., Schaffer M., (2009): Organizational readiness for evidence-based practice, The Journal of nursing administration, $\operatorname{vol}(39), \operatorname{No}(2)$ Pp.91-7.

13. Ghoneim M., Alaa Elden S., Okab M., Elsaay O., (2012): Impact of Implementing Nursing Care Protocol on Moderate Head Injured Patient's Outcom, Journal of American Science, vol 8, No (7) Pp.649-64.

14. Glacken M., Chaney D., (2004): Perceived barriers and facilitators to implementing research findings in the Irish practice setting, Journal of clinical nursing, vol (13), No (6): Pp.731-40.

15. Holleman G., Eliens A., van Vliet M., van Achterberg T., (2006): Promotion of evidencebased practice by professional nursing associations, Journal of advanced nursing, vol 53, No (6) Pp.702-9.

16. Hesdorffer D., Ghajar J., Iacono L., Predictors of compliance with the evidencebased guidelines for traumatic brain injury care: a survey of United States trauma centers. (2002): The Journal of trauma, vol 52, No (6) Pp.1202-9.

17. Hesdorffer D., Ghajar J., (2007): Marked improvement in adherence to traumatic brain injury guidelines in United States trauma centers, The Journal of trauma. vol (63), No (4) Pp.8417.

18. Kirkness C., Burr R., Cain K., Newell D., Mitchell P., (2005): Relationship of cerebral perfusion pressure levels to outcome in traumatic brain injury, Acta Neurochir Suppl, vol (95), No(1) Pp.13-6.

19. Ladanyi S., Elliott D., (2008): Traumatic brain injury: an integrated clinical case presentation and literature review part II: the continuum of care, Australian critical care : official journal of the Confederation of Australian Critical Care Nurses, vol 21, No (3) Pp.141-53.

20. Lemke D., (2007): Sympathetic storming after severe traumatic brain injury. Critical care nurse, vol 27, No (1) Pp.30-7.

21. March K., Wellwood J., Lovasick D., Madden L., Criddle L., Henrickson S. (2004): Craniocerebral trauma In: Bader M, Littlejohns 
L. AANN Core Curriculum for Neuroscience Nurses, $4^{\text {th }}$ ed, St Louis: Saunders Pp. 277-334.

22. Montaser T., Hassan A., (2013): Epidemiology of moderate and severe traumatic brain injury in Cairo University Hospital in 2010, Critical Care, vol 17, No (2) Pp.320-21.

23. Morton P., Fontaine D., (2009): Critical care nursing; a holistic approach, $9^{\text {th }}$ ed, Philadelphia: Lippincott Williams \& Wilkins Pp. 839-60.

24. McCaughan D., Thompson C., Cullum N., Sheldon T., Thompson D., (2002): Acute care nurses' perceptions of barriers to using research information in clinical decision-making., Journal of advanced nursing, vol (39), No (1): Pp.46-60.

25. McNett M., Gianakis A., (2010): Nursing interventions for critically ill traumatic brain injury patients. The Journal of neuroscience nursing, journal of the American Association of Neuroscience Nurses, vol 42, No (2) Pp.71-7; quiz 8-9.

26. Majid S., Foo S., Luyt B., Zhang X., Theng Y., Chang Y., (2011): Adopting evidence-based practice in clinical decision making:nurses' perceptions, knowledge, and barriers, J Med Libr Assoc vol(23), No(8) Pp.99.

27. Mehrdad N., Salsali M., Kazemnejad A., (2008): The spectrum of barriers to and facilitators of research utilization in Iranian nursing, Journal of clinical nursing, vol (17), No (16) Pp.2194-202.

28. Oh E., (2008): Research activities and perceptions of barriers to research utilization among critical care nurses in Korea. Intensive \& critical care nursing : the official journal of the British Association of Critical Care Nurses, vol (24), No (5) Pp.314-22.

29. Rosenfeld J., Maas A., Bragge P., MorgantiKossmann M., Manley G., Gruen R., (2012): Early management of severe traumatic brain injury, Lancet, vol (380), No (9847) Pp.1088-98.

30. Ryan D., (2009): Caring for patients with traumatic brain injuries: Are you up to the challenge?, American Nurse Today, vol 4, No (8) Pp.19-22.

31. Susman M., DiRusso S., Sullivan T., Risucci D., Nealon P., Cuff S. (2002): Traumatic brain injury in the elderly: increased mortality and worse functional outcome at discharge despite lower injury severity, The Journal of Trauma, vol 53, No (2) Pp.219-23

32. Sharaf I., (1999): Road traffic accidents in Egypt (road war). Al Ahram daily news March 6 \& (1999).

33. Stein S., Georgoff P., Meghan S., Mirza K., El Falaky O., (2010): Relationship of aggressive monitoring and treatment to improved outcomes in severe traumatic brain injury, Journal of neurosurgery, vol 112, No (5) Pp.1105-12.

34. Selassie A., Zaloshnja E., Langlois J., Miller T., Jones P., Steiner C., (2008): Incidence of long-term disability following traumatic brain injury hospitalization, United States, 2003. J Head Trauma Rehabil, vol (23), No(6) Pp.12331.

35. Tagliaferri F., Compagnone C., Korsic M., Kraus J., (2006): Asystematic review of brain injury epidemiology in Europe, ActaNeurochir, vol 148, No(3) Pp.255-68.

36. Thompson H. Clinical practice guideline series update. (2011) journal of the American Association of Neuroscience Nurses, vol 43, No (4) Pp.233-5.

37. Tan M., Akgun Sahin Z., Kardas Ozdemir F., (2012): Barriers of research utilization from the perspective of nurses in Eastern Turkey, Nursing outlook, vol (60), No (1) Pp.44-50.

38. Tsai S., (2000): Nurses' participation and utilization of research in the Republic of China, International journal of nursing studies, vol(37), No(5) Pp. 435-44.

39. Uysal A., Temel A., Ardahan M., Ozkahraman S., (2010): Barriers to research utilisation among nurses in Turkey, Journal of clinical nursing, vol(19), No(23) Pp.3443-52.

40. Wang X., Dong Y., Han X., Qi X., Huang C., Hou L., (2013): Nutritional support for patients sustaining traumatic brain injury: a systematic review and meta-analysis of prospective studies. PlOS one, vol 8, No (3)Pp.58-83. 